\title{
Um Veículo Aéreo Não Tripulado para Encontrar Focos de Poluição
}

\author{
Marcelo Maia da Silva ${ }^{1}$, Heitor Freitas ${ }^{2}$, Bruno Faiçal ${ }^{2}$, \\ Gerson Serejo $^{3}$, Jó Ueyama ${ }^{2}$, Gustavo Pessin ${ }^{1,3}$ \\ ${ }^{1}$ Instituto de Ciências Exatas e Naturais \\ Universidade Federal do Pará (UFPA) - Belém, PA, Brasil \\ ${ }^{2}$ Instituto de Ciências Matemáticas e de Computação (ICMC) \\ Universidade de São Paulo (USP) - São Carlos, SP, Brasil \\ ${ }^{3}$ Laboratório de Computação Aplicada \\ Instituto Tecnológico Vale - Belém, PA, Brasil \\ marcelomaia@ufpa.br, \{heitorfv,bsfaical, joueyama\}@icmc.usp.br \\ gerson.serejo@pq.itv.org, gustavo.pessin@itv.org
}

Resumo. O presente trabalho tem como objetivo o desenvolvimento de um Veículo Aéreo Não Tripulado (VANT) para encontrar focos de poluição. Um protótipo de baixo custo foi construído para obtenção de concentração de poluentes (CO) e variáveis ambientais (umidade e temperatura) e duas metodologias de coleta em voo foram empregadas para coleta dos dados. Os testes de coleta foram realizados na área do Campus 2 da USP/ICMC.Como resultado, foi identificada a área com maior concentração de calor por meio de um algoritmo de interpolação. Por fim, notou-se que o protótipo de baixo custo foi capaz de monitorar poluentes atmosféricos e variáveis ambientais. Nota-se que o modelo serve também para outros tipos de medições como de agrotóxicos e radiações. Como contribuição deste trabalho, tem-se uma alternativa barata para monitoramento de poluentes direcionadas para contextos mais específicos.

\section{Introdução}

Normalmente, estações de monitoramento de qualidade do ar tipicamente instaladas por agências de proteção ao meio ambiente são equipamentos caros, localizados em posições fixas e responsáveis por monitorar grandes áreas [Devarakonda et al. 2013]. Entretanto, veículos aéreos não tripulados (VANTs) podem ser empregados como estações móveis, a fim de permitir a percepção de variáveis de interesse em regiões mais específicas. Além disso, VANTs podem ser mais baratos e apresentam flexibilidade no planejamento do voo com uma maior possibilidade de aproximação a um alvo de interesse [Yan et al. 2010]. Este trabalho propõe uma forma barata e flexível de análise de qualidade de ar, onde dois modelos de coleta (que são complementares) são propostos para encontrar focos de poluição. Com o uso do kit móvel proposto neste trabalho, coletas e análises para o entendimento dos padrões das poluições serão facilitadas para diversas localidades e contextos. O kit apresentado é um dispositivo de computação móvel transportado num VANT, que é composto por sensores que coletam variáveis climáticas (como temperatura e umidade) e poluentes atmosféricos (CO).

Este trabalho tem a seguinte estrutura: a Seção 2 apresenta uma revisão da literatura na área de qualidade de ar e VANTs, visando relacionar trabalhos da literatura 
com este; na Seção 3, é descrito o modelo proposto para o monitoramento, detalhando o protótipo de hardware, software e as metodologias de coleta. Na sequência, a Seção 5 apresenta os resultados alcançados e, finalmente, na Seção 6, são feitas as considerações finais e os trabalhos futuros.

\section{Trabalhos Relacionados}

Nesta Seção são apresentados trabalhos que usam VANT, com a finalidade de comparálos à esse trabalho. Por fim, é apresentada uma discussão sobre os trabalhos correlatos. Faiçal et al. [Faiçal et al. 2014] descrevem uma arquitetura orientada ao problema de auto ajuste de rotas do VANT ao pulverizar produtos químicos em uma plantação. Onde um algoritmo é proposto e avaliado (com o uso de algoritmo de agrupamento DBSCAN), considerando o retorno de informação oriunda de uma rede de sensores sem fio simulada, variações de vento em força e direção, observou-se que a arquitetura em questão pode notavelmente reduzir o desperdício de pesticidas e fertilizantes. São evidenciados na Figura 1 os testes feitos em simulação, onde foram usados algoritmos de agrupamento para determinar os locais adequados para aplicação de pesticida.

Roldán et al. [Roldán et al. 2015] criaram e validaram uma plataforma sensorial móvel para monitoramento de estufas. A plataforma consiste um conjunto de sensores acoplados a um quadrotor, os objetivos desse sistema incluem capturar medidas de concentração de temperatura, umidade, luminosidade e $\mathrm{CO}_{2}$ e gerar mapas dessas variáveis. O posicionamento dos sensores foram determinados por meio de um estudo de aerodinâmica do quadrotor e a influência dos fluxos de ar e de seus motores. A contribuição primária do trabalho é a determinação do lugar ótimo para cada sensor, considerando o fluxo de ar. No trabalho em questão, foi mapeada a concentração de calor dentro de uma estufa como mostra a Figura 2.

Caltabiano et al. [Caltabiano et al. 2005] conduziram o projeto ROBOVOLC para exploração e análise de fenômeno vulcânico sem nenhuma interação humana. O veículo aéreo não tripulado teve de ser autônomo. Devido a duas dificuldades: (i) distância segura da pluma (nuvem) vulcânica e (ii) dificuldade de identificação visual do veículo.

\subsection{Discussão sobre os trabalhos relacionados}

Observando os trabalhos relacionados, verifica-se que análises de qualidade do ar com sensores e mapeamentos têm sido implementados, contudo os estudos apresentados possuem lacunas. Faiçal et al. [Faiçal et al. 2014] implementaram uma arquitetura de auto ajuste de rota de VANT para pulverização de produtos químicos, com geração de mapas de concentração de pesticidas, todavia, a validação da arquitetura foi em ambiente simulado. Roldán et al. [Roldán et al. 2015] implementaram uma plataforma sensorial para estufas, definiram através de simulação os locais ótimos dos sensores em um quadrotor, contudo, o experimento não foi realizado considerando as variações de altura. Caltabiano et al. [Caltabiano et al. 2005], implementaram um VANT para análise de pluma vulcânica, porém, não foram implementados mapas de concentração de poluentes. Por estas razões, este trabalho implementa um estudo em campo com dados reais coletados por um VANT, com a finalidade de encontrar poluição em uma determinada área ou altura. 


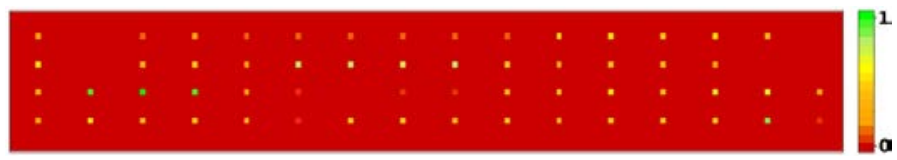

(a)

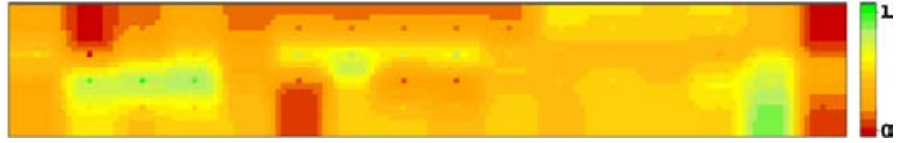

(b)

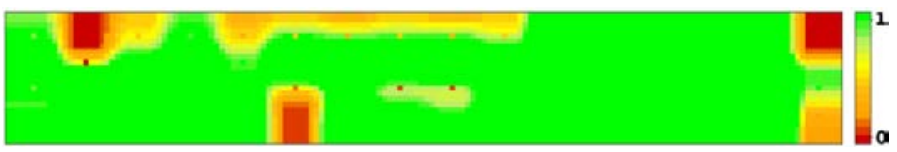

(c)

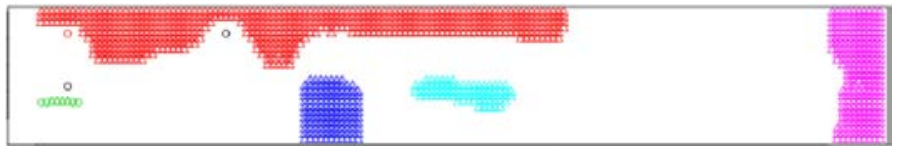

(d)

Figure 1. (a) Simulação de um campo de plantação representado numa matriz 2D apresentado por [Faiçal et al. 2014]. A variação de vermelho para verde significa menos pesticidas recebidos. A maior parte da área está vermelha por que os sensores são pontuais. A distribuição de pesticidas está desigual devido à variação de vento na simulação. (b) Mapa após aplicação de técnica de interpolação. (c) Mapa após a aplicação de um limiar, que determina onde há menos pesticida. (d) Identificação por agrupamento usando o algoritmo DBSCAN .

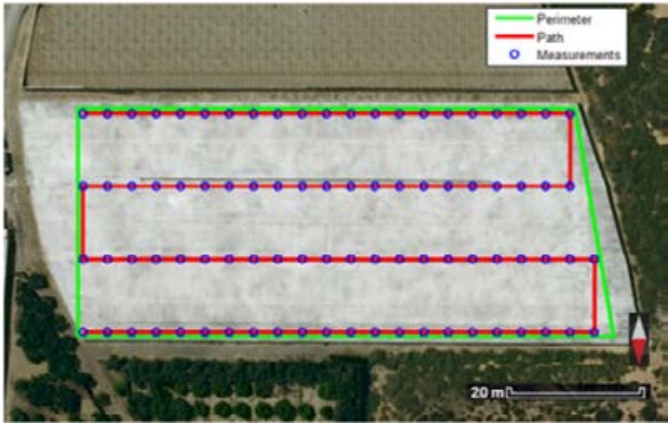

(a)

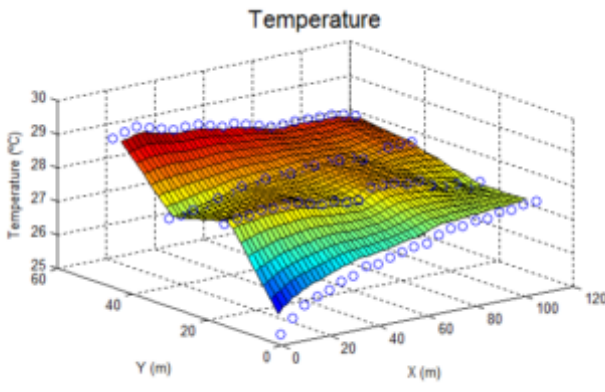

(b)

Figure 2. (a) $O$ caminho de coleta do quadrotor dentro da estufa, os pontos no traçado são os locais de coleta. (b) Mapa de temperatura dentro da estufa [Roldán et al. 2015] 


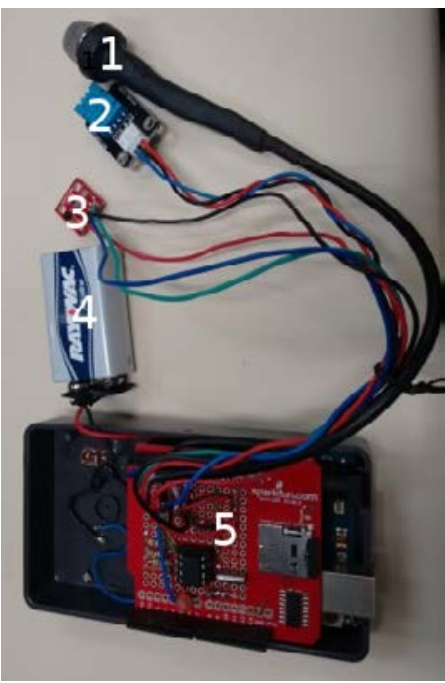

(a)

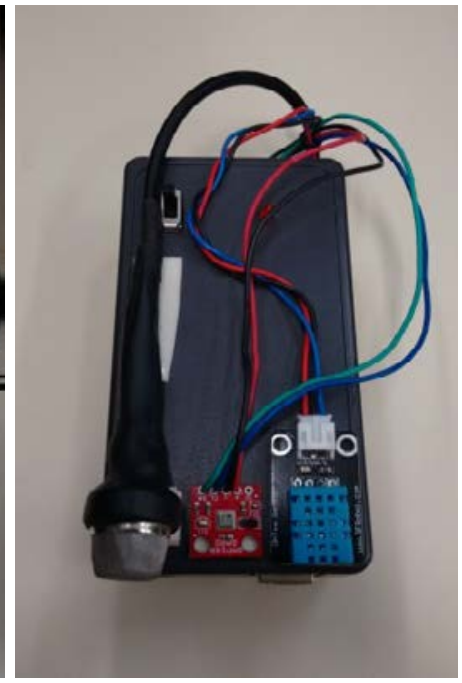

(b)

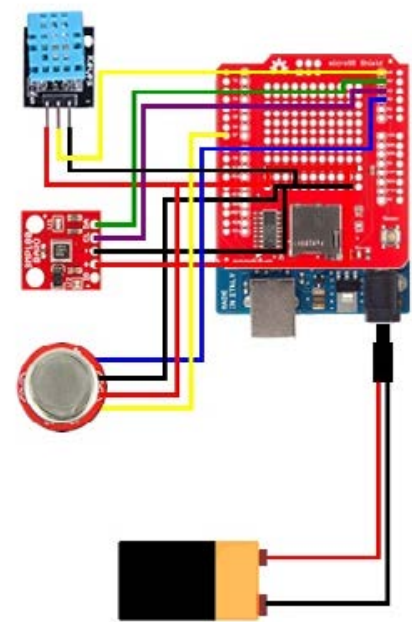

(c)

Figure 3. (a) Protótipo de coleta de CO: (1) detector de CO MQ-2, (2) sensor de temperatura e umidade DHT11, (3) barômetro BMP180 (4) bateria 9v e (5) Arduino Uno, DS1307 e cristal 32.768 usados para o relógio do Arduino e SD-card. (b) Protótipo fechado. (c) Esquema de circuito do protótipo.

\section{Materiais e métodos}

Foram implementados um protótipo de baixo custo e duas metodologias de coletas, ambos descritos a seguir.

\subsection{Protótipo}

O protótipo de coleta de poluentes e variáveis ambientais de baixo custo (Figura 3), consiste de um Arduino UNO, dois possíveis sensores de monóxido de carbono, sendo MQ-2 e MQ-7, um sensor de umidade e temperatura DHT11, um sensor de pressão e temperatura BMP180, um shield SD, um timer para ter acesso à hora e por fim o software de controle do protótipo. Esse protótipo móvel foi acoplado a um VANT (Figura 4), em colaboração com o ICMC-USP.

A Figura 4(c) mostra o VANT MikroKopter Okto $^{1}$ com vista de cima, o módulo UAV Brain que é o dispositivo computacional do VANT é exposto na Figura 5 com detalhes de sua implementação. O ZigBee, das Figuras 5(a)i e 5(a)ii, é o responsável pela comunicação sem fio, o Arduino, em conjunto com a Power Meter Shield (Figuras 5(b)ii e 5(b)iv respectivamente), atuam no monitoramento do consumo de energia elétrica do ZigBee, há um sensor DHT22 de temperatura e umidade para registro de dados climáticos 5(c)v. O Raspberry Pi, exposto na Figura 5(b)iii, é o núcleo do agente computacional UAV Brain, ao qual são delegadas as funções computacionais do VANT como registro de logs. O objetido da integração do VANT com o protótipo móvel de coleta é implementar dois modelos de coleta: (i) zigue-zague e (ii) subida/descida, os quais serão explicados a seguir.

\footnotetext{
${ }^{1} \mathrm{O}$ VANT utilizado neste trabalho foi disponibilizado pelo pesquisador colaborador desta pesquisa Jó Ueyama, do ICMC-USP. O mesmo foi utilizado durante uma visita técnica a USP entre 15/06 até 04/07 de 2015.
} 


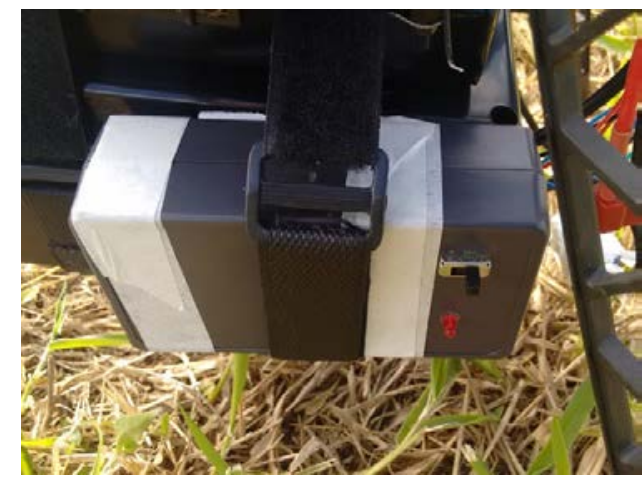

(a)

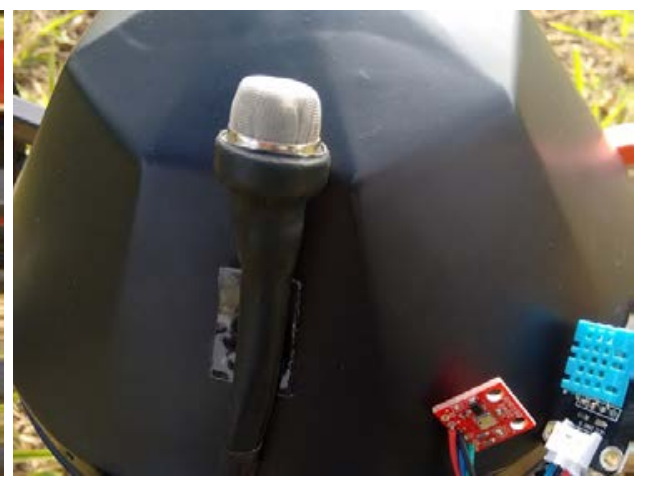

(b)

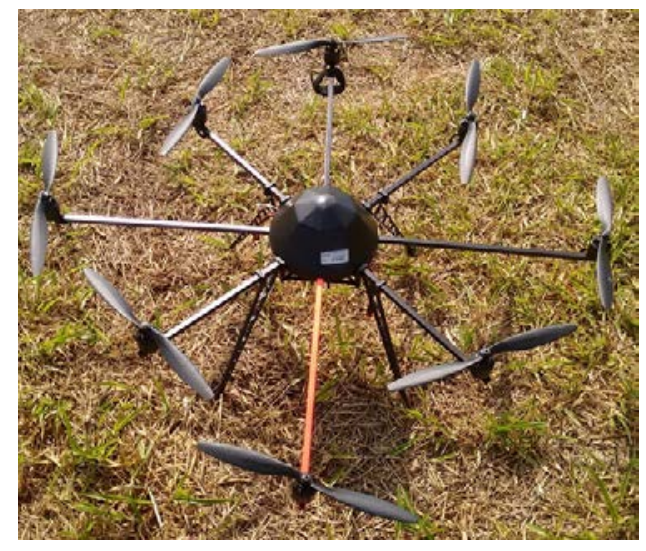

(c)

Figure 4. (a) $O$ controlador Arduino em uma caixa de proteção. (b) Sensores MQ-2, BMP180 e DHT11 (esquerda para direita). (c) VANT MikroKopter Okto, com oito hélices classe III.

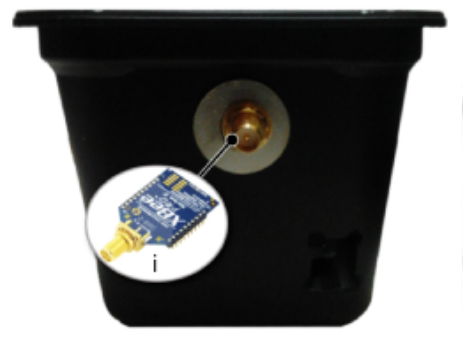

(a)

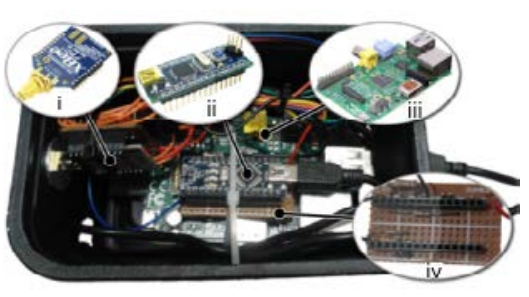

(b)

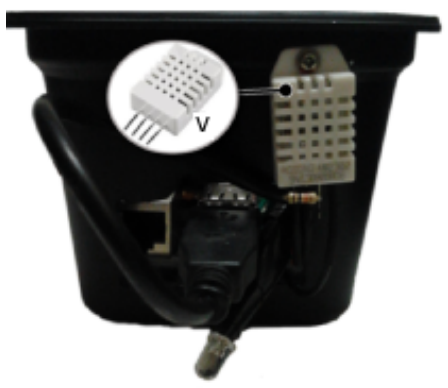

(c)

Figure 5. (a) Vista frontal. (b) Vista superior. (i) Rádio transceptor ZigBee. (ii) Arduino Nano. (iii) Raspberry Pi. (iv) Power Meter Shield [Ueyama et al. 2014]. 


\section{Metodologia de coleta e análise empregada em plataforma móvel (VANT)}

Foram elaborados dois planos de voo: (i) zigue-zague e (ii) variação de altura, o modelo de coleta em zigue-zague consiste de um voo em forma de zigue-zague em uma altura fixa a fim de coletar dados em uma determinada área. O modelo de subida/descida consiste de subir ou descer com paradas periódicas para coleta de dados, a fim de detectar as maiores concentrações de poluentes em uma determinada altura. Foi verificado por [Roldán et al. 2015] que o melhor lugar para a localização dos sensores que sofrem com a influência de vento é no topo do centro do VANT, desta forma, é indicado que sensores de gases, temperatura e umidade fiquem nesta posição. O sensores de temperatura e umidade devem ficar afastados de fontes de calor, como o sensor de CO MQ-2 (mostrado no centro da Figura 4(b)) que aquece. Este método de coleta tem como objetivo realizar reconhecimento de concentração de poluentes ou variáveis climáticas. Após um voo inicial, o VANT pode indicar uma altura ou uma área específica onde deve-se realizar um outro voo de reconhecimento. O modelo propõe mapear e encontrar focos de poluição, contudo, observa-se que além de poluentes podem ser medidos quaisquer variáveis, como pesticidas e radiações, desde que sejam consideradas as interferências de fatores durante o voo, como vento, calor, umidade, dentre outros, nas leituras dos sensores.

\section{Experimentos e resultados}

Os dois modelos de coleta (zigue-zague e com variação de altura) foram implementados usando o dispositivo móvel acoplado ao VANT, devido a necessidade de realizar os voos afastados de um centro urbano (pois o VANT representa risco de morte). As variações de CO foram mínimas, desta forma, foram analisados os dados de temperatura, especificamente com o sensor BMP180, pois, houveram variações de temperatura ao longo do voo. A taxa de leitura do sensor BMP180 é $12 \mathrm{~Hz}$, como a velocidade média do VANT nestas coletas foram de $3 \mathrm{~m} / \mathrm{s}$ o sensor BMP180 registrou na média 4 leituras a cada metro, que possibilitou uma boa amostragem por espaço.

\subsection{Coleta em zigue-zague}

A coleta (i) zigue-zague destacada na Figura 6, foi realizada em uma altura fixa de $25 \mathrm{~m}$ para cobrir uma área de $1600 \mathrm{~m}^{2}$ percorrendo $1039 \mathrm{~m}$ em $5 \mathrm{~min}$ e $46 \mathrm{~s}$. Foram desconsiderados os dados coletados durante a subida e descida, pois não faziam parte deste modelo de coleta. Com os dados obtidos, que são pontos de latitude, longitude com as medidas de $\mathrm{CO}$, temperatura e umidade para cada ponto, foi aplicado um método de interpolação linear $^{2}$, objetivando encontrar concentrações de dados coletados, neste caso a temperatura. Como resultado desta interpolação dos dados, foi obtido o gráfico da Figura 6(a), o qual mostra as concentrações de temperatura (maiores temperaturas tendem ao vermelho e menores ao azul). Voos em sequência podem ser realizados em áreas específicas, com uma maior densidade de pontos em um menor espaço, com o objetivo de mapear e entender com maior grau de resolução poluentes/variáveis climáticas de uma determinada área.

\footnotetext{
${ }^{2}$ Algoritmo de interpolação linear, especificamente o método LinearNDInterpolator() do pacote scipy da linguagem Python. A interpolação é construída por triangular os dados de entrada com Qhull e, em cada triângulo realiza uma interpolação baricêntrica linear.
} 


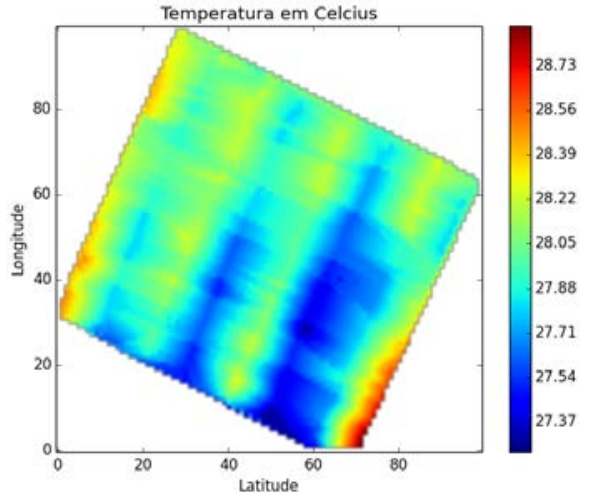

(a)

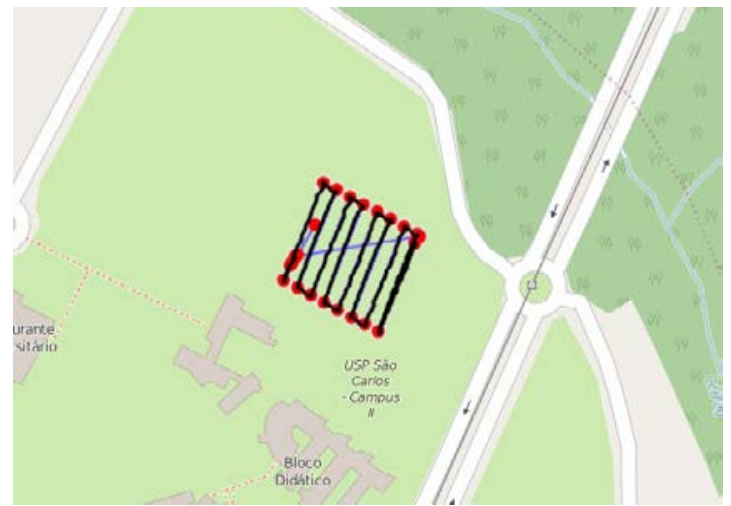

(b)

Figure 6. (a) Mapa de concentração de temperatura com o sensor BMP180. (b) USP São Carlos campus 2, área onde foi realizada a coleta e o conjunto de waypoints usados pelo VANT em conjunto com seu software de controle para realizar a coleta.

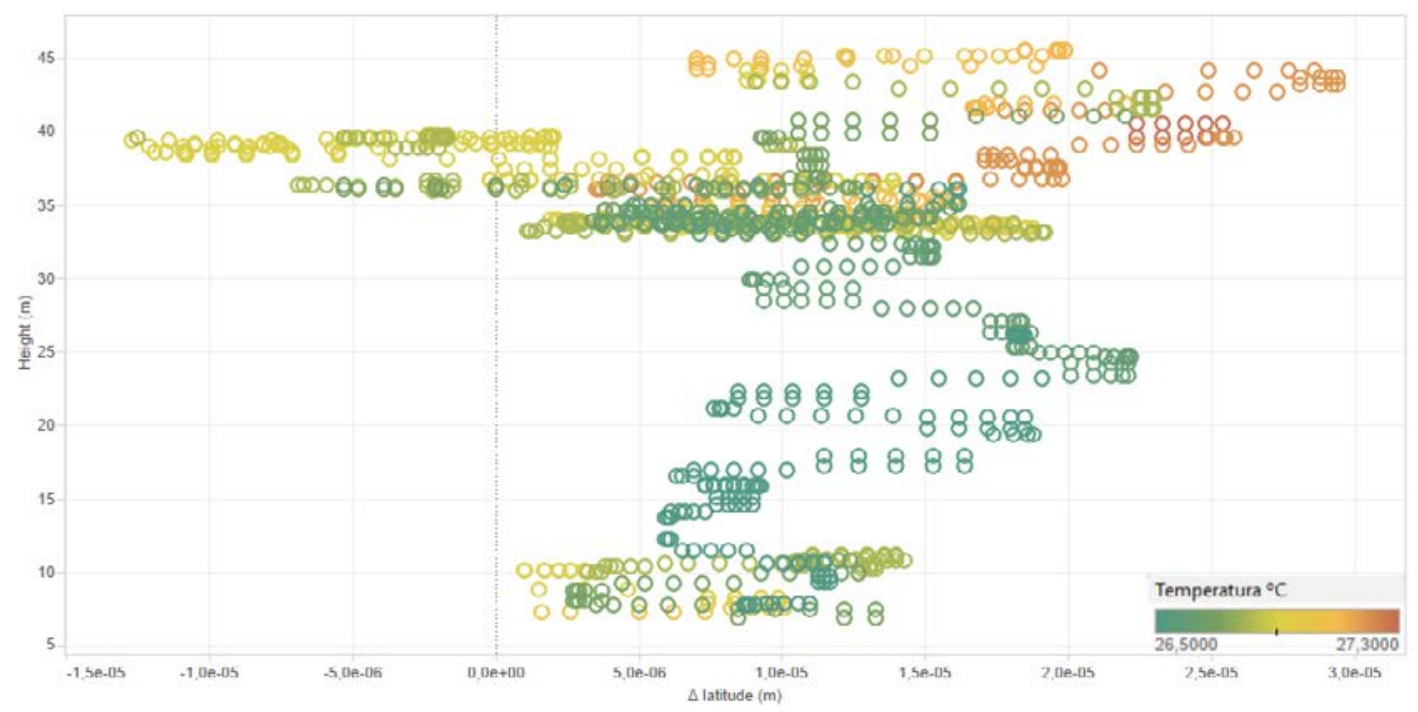

Figure 7. Dados coletados de temperatura considerando a variação de altura no ICMC campus 2. A cor dos pontos indicam a temperatura num dado ponto de coleta. $O$ eixo x é a variação do VANT em relação ao seu ponto inicial na latitude, o eixo y diz respeito a altura. Podemos ver que a concentração de temperatura mostrou-se maior na altura de $\mathbf{4 0} \mathrm{m}$, também que a variação em torno do eixo $\mathrm{x}$ foi maior devido ao vento ser mais forte de acordo com a altura. 


\subsection{Coleta com variação de altura}

A coleta (ii) com variação de altura evidenciada na Figura 7 foi realizada com uma variação de $5 \mathrm{~m}$ a $40 \mathrm{~m}$ de altura num total de 2417 leituras em $3.3 \mathrm{~min}$, onde a cada $10 \mathrm{~m}$ houve uma parada de $30 \mathrm{~s}$ para coletar dados de temperatura. O eixo x é referente ao desvio do VANT na latitude em relação ao seu ponto inicial, o eixo y é referente à altura. Nesta coleta foi usado o sensor BMP180 para medir as variações de altura e temperatura ao longo do voo, percebe-se que a concentração de temperatura e a variação em torno do eixo x mostraram-se maiores na altura de $40 \mathrm{~m}$ (a variação em torno do eixo x é maior em função do vento). O VANT poderia ser então empregado para encontrar a altura que apresenta maior concentração de poluentes e a partir disso realizar o voo em zigue-zague. Com este modelo de coleta é possível ter uma alternativa aos balões meteorológicos, onde o VANT apresenta uma flexibilidade de voo que o balão não possui.

\section{Considerações finais e trabalhos futuros}

Neste estudo foi proposto um protótipo móvel de baixo custo para análise de qualidade de ar, objetivando entender as variações de poluentes em diferentes contextos. $\mathrm{O}$ protótipo foi acoplado a um VANT e testes com dois métodos de leitura foram realizados. Informações sobre monitoramento de qualidade de ar não são fáceis de serem obtidas; e mesmo onde há monitoramento, como se pode ver nos relatório da CETESB [CETESB 2014], as análises são feitas em resolução de cidade/bairro. Generalizando um amplo espaço, são análises que não apresentam refinamento de localização. Comparando nosso trabalho com a abordagem tradicional, aplicada por agências ambientais oficiais, $o$ presente trabalho mostrou a capacidade de monitorar os poluentes em áreas de interesse mais específicos de forma mais prática.

Como resultados, foram realizadas duas caracterizações de uma localização dentro do ICMC Campus 2, onde foram analisadas as concentrações de temperatura (não houve variação de $\mathrm{CO}$ neste ambiente de coleta), como dito anteriormente. O modelo proposto pode servir para encontrar concentração de poluentes, agrotóxicos, radiação, ilhas de calor, etc. Uma observação importante é que se deve considerar o posicionamento dos sensores e os fatores durante o voo que podem afetar em suas leituras.

\subsection{Trabalhos Futuros}

Diversas outras atividades ainda são previstas, entre elas, a integração do protótipo móvel de coleta com GPS (usou-se o GPS do VANT), análises em tempo real para encontrar focos de poluição em tempo de voo e a aquisição de outros sensores como de pesticidas, radiação, e de material particulado. Diversos são os pontos em abertos neste tipo de pesquisa. Em termos de inovação científica, existe um vasto campo a ser explorado na área, tanto em desenvolvimento de hardware como no desenvolvimento de software. Em termos de monitoramento ambiental, a pesquisa pode ter um desdobramento mais específico, como no mapeamento de concentração de pesticidas, de material particulado e até de radiação.

\section{Agradecimentos}

Os autores gostariam de agradecer aos colegas Bruno Ferreira (UFPA), Eduardo Carvalho (ITV), Leon Cardoso (ITV) e Geraldo Pereira (USP) por inspirações e auxílios no desen- 
volvimento do trabalho. Ainda, os autores gostariam de agradecer ao apoio financeiro recebido através da Chamada 59/2013 MCTI/CT-Info/CNPq, processo 440880/2013-0.

\section{References}

[Caltabiano et al. 2005] Caltabiano, D., Muscato, G., Elettrica, I., Doria, V. A., Federico, C., Giudice, G., Palermo, S., and Malfa, V. U. L. (2005). Architecture of a UAV for volcanic gas sampling. 1:739-744.

[CETESB 2014] CETESB (2014). Relatorio de qualidade do ar no estado de sao paulo.

[Devarakonda et al. 2013] Devarakonda, S., Sevusu, P., Liu, H., Liu, R., Iftode, L., and Nath, B. (2013). Real-time Air Quality Monitoring Through Mobile Sensing in Metropolitan Areas.

[Faiçal et al. 2014] Faiçal, B. S., Costa, F. G., Pessin, G., Ueyama, J., Freitas, H., Colombo, A., Fini, P. H., Villas, L., Osório, F. S., Vargas, P. a., and Braun, T. (2014). The use of unmanned aerial vehicles and wireless sensor networks for spraying pesticides. Journal of Systems Architecture, 60(4):393-404.

[Roldán et al. 2015] Roldán, J., Joossen, G., Sanz, D., del Cerro, J., and Barrientos, A. (2015). Mini-UAV Based Sensory System for Measuring Environmental Variables in Greenhouses. Sensors, 15(2):3334-3350.

[Ueyama et al. 2014] Ueyama, J., Freitas, H., Faical, B. S., Filho, G. P., Fini, P., Pessin, G., Gomes, P. H., and Villas, L. A. (2014). Exploiting the use of unmanned aerial vehicles to provide resilience in wireless sensor networks. IEEE Communications Magazine, 52(12):81-87.

[Yan et al. 2010] Yan, L., Gou, Z., and Duan, Y. (2010). A uav remote sensing system: Design and tests. In Geospatial Technology for Earth Observation, pages 27-44. Springer. 